\title{
Synthesis and Biological Activity of Some New Substituted Quinazolin-4(3H)-Ones
}

\author{
Nadia T.A. Dawood, Nahed F. Abdel-Ghaffar and Fekria \\ M.A. Soliman* \\ Department of Chemistry, Faculty of Science "Girls", Al- \\ Azhar University, Cairo, Egypt.
}

\begin{abstract}
3 - (4-AMINOPHENYL)-2-benzyl-4-oxo-3,4-dihydroquinazoline 3 1a and 3 - (4 - aminophenyl) - 2 - benzyl - 6 - bromo - 4 - oxo 3,4 dihydroquinazoline $1 \mathrm{~b}$ were prepared and diazotized by nitrous acid to give " $2 a, b$ ". The diazonium chloride derivatives were converted to the corresponding 3-arylhydrazono derivatives on treatment with active methylene compounds namely acetylacetone, ethyl acetoacetate and/or ethyl cyanoacetate. The hydrazono derivatives were converted to the corresponding pyrimidino derivatives "6a- $\mathrm{f}$ ", pyrazolo derivatives "7a-d" and pyrazoline derivatives "8a-d" and "9a-f" in order to study their antimicrobial activity. A significant level of activity of the new products was observed.
\end{abstract}

Keywords: 2- Benzyl - 4(3H) quinazolinone - 4 - (3H) - one, 6-Bromo derivative, Aryl hydrazones, $\beta$-Diketones and Biological activity.

Quinazolinone derivatives attract a widespread interest due to their biological activities ${ }^{(1)}$. They are pharmaceutically important as antifibrillatory, choleretic, antiphlogistic $^{(2)}$, and anticonvulsant agents ${ }^{(3)}$. They have also been successfully tested as inhibitors of the DNA repair enzyme poly (ADP-ribose) polymerase ${ }^{(4)}$ and for their antineoplastic activity ${ }^{(5)}$. It is well known that both pyrimidine ${ }^{(6-8)}$ and pyrazole ${ }^{(9-11)}$ moieties possess various therapeutic activities.

The formation of 2-alkyl-4 $(3 \mathrm{H})$ quinazolinones by condensation of anthranilic acid or substituted anthranilic acid and amides is designated as the Niementowski reaction ${ }^{(12)}$. Ziegler, Steiger and Kappe ${ }^{(13)}$ also demonstrated the formation of 2-alkyl, 2-aralkyl and 2-aryl-4(3H) quinazolinones in moderate yields from isatoic anhydride as the source of the anthraniloyl group and the corresponding carboxamide. In view of these and in continuation of our earlier work on substituted-quinazolin-4(3H)-ones ${ }^{(14-17)}$, it was thought of interest to combine the pharmacophoric groups (pyrimidine, prazoline) as well as quinazoline moiety. The new compounds have been synthesized and screened for their antimicrobial activities.

\footnotetext{
"Author to whom correspondence should be addressed E-mail:

kachef_hicham@yahoo.com
} 


\section{Results and Discussion}

3- (4-aminophenyl) - 2 - benzyl -4 - oxo -3, 4- dihydroquinazoline" $1 \mathrm{a}^{\prime \prime}$ and 3(4-aminophenyl) - 2 - benzyl - 6 - bromo - 4 - oxo - 3, 4-dihydroquinazoline " $1 b^{\prime \prime}$ were prepared according to the literature procedures ${ }^{(18-20)}$. These derivatives which bear a bulky substituent at position-2 ${ }^{(14,16)}$. $($.f. Fig 1).

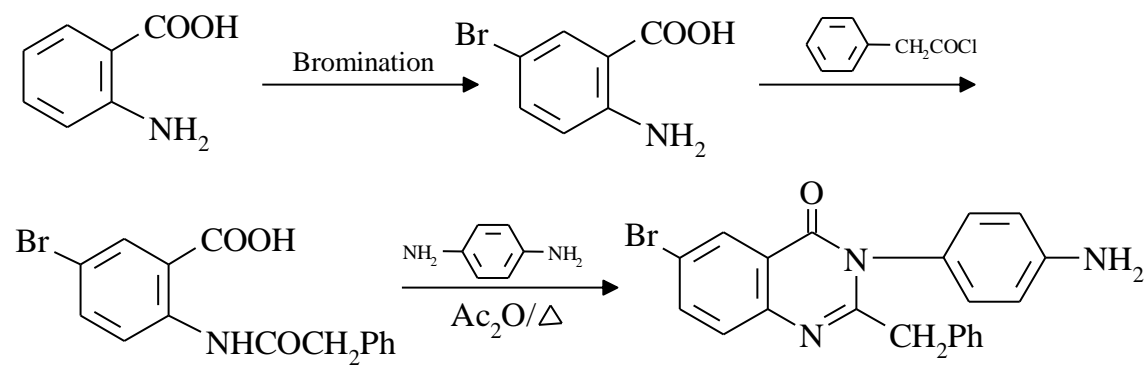

Fig. 1.

(1b)

Compounds " $1 \mathrm{a}, \mathrm{b}$ " were subjected to diazotization with nitrous acid $\left(\mathrm{NaNO}_{2}\right.$ and $\mathrm{HCl}$ ) at $0-5^{\circ} \mathrm{C}$ to give 4-(2-benzyl-4-oxo-3, 4-dihydroquinazolin-3-yl) benzene diazonium chloride "2a" and 4-(2-benzyl-6-bromo-4-oxo-3, 4-dihydroquinazolin-3-yl) benzene diazonium chloride " $2 \mathrm{~b}$ ". Treatment of " $2 \mathrm{a}, \mathrm{b}$ " with $\beta$ diketones namely, acetylacetone, and ethylacetoacetate or active methylene compounds such as ethyl cyanoacetate in boiling ethanol containing fused sodium acetate afforded the corresponding 3-\{4-(2-benzyl-4-oxo-3,4dihydroquinazlin-3-yl) phenylhydrazono\}pentane-2,4-diones "3a", 3-\{4-(2benzyl-6-bromo-4-oxo-3,4dihydroquinazlin-3-yl)phenyl hydrazono\}pentane2,4-diones"3b",2-\{4-(2-benzyl-4-oxo-3,4-dihydroquinazlin-3-yl) phenylhydrazono $\}$ 3-oxobutanoic acid ethyl esters "4a,b" and 2-\{-4-(2-benzyl-4-oxo-3,4dihydroquinazlin-3-yl)phenylhydrazono $\}$-3-cyanoaceticc acid ethyl esters " $5 \mathrm{a}, \mathrm{b}$ ", respectively. The IR spectrum of 3 a agreed well with the proposed structure ${ }^{(18)}$ The mass spectra of compounds which contain bromine atoms $3 \mathrm{~b}, 4 \mathrm{~b}$ and $5 \mathrm{~b}$ showed fragments corresponding to the typical bromine isotopes $\left({ }^{79} \mathrm{Br}\right.$ and $\left.{ }^{81} \mathrm{Br}\right)$ patterns.

Interaction of the hydrazono derivatives " $3 \mathrm{a}, \mathrm{b}$ " with guanidine hydrochloride, thiourea and urea in refluxing ethanol afforded the cyclized products 2 - benzyl - 3 - \{4-(2- amino-4,6-dimethyl $\}$ - 2, 5 - dihydropyrimidin-5ylildine) hydrazinophenyl $\}$ - 4-oxoquinazolines "6a,d", 6-bromo-2-benzyl-3-\{4(2 - imino - 4, 6 - dimetyyl - 2 - thioxopyrimidin - 5 - ylildine) hydrazinophenyl $\}$ 4-oxoquinazolines "6b-e" and 2 - benzyl - 3 - \{4 - (2 - imino - 4, 6 - dimetyyl -2oxopyrimidin-5-ylildine) hydrazinophenyl $\}-4$-oxoquinazolines "6c, $\mathrm{f}^{\prime}$. This is in agreement with the previous findings ${ }^{(21,22)}$. 
Similarly, the hydrazonoquinazolone derivatives "3a, b" reacted with phenylhydrazine and 4-nitrophenyl hydrazine in boiling acetic acid containing sodium acetate giving the corresponding2-benzyl-3-\{4-(3,5-dimethyl-1-phenyl1-H-pyrazol-4-yl)azophenyl\}-4-oxoquinazolines "7a", 2-benzyl-6-bromo-3-\{4(3,5-dimethyl-1-phenyl-1H-pyrazol-4-yl)-4-oxoquinazolines "7b" , 2-benzyl-3\{4-(3,5-dimethyl-1(-4-nitrophenyl-1H- pyrazol-4-yl)-4-oxoquinazolines "7c" and 2 - benzyl - 6-bromo - 3 -\{4-(3,5-dimethyl - 1 (-4-nitrophenyl-1H- pyrazol-4-yl) 4-oxoquinazolines " $7 \mathrm{~d}$ ", respectively via thermal cyclization.

Interaction of the substituted hydrazono quinazolone derivatives " $4 \mathrm{a}, \mathrm{b}$ " with hydrazines, namely, hydrazine hydrate, phenyl hydrazine and 4nitrophenylhydrazine in boiling ethanol afforded the corresponding 2-benzyl-3\{4- (3-methyl - 5 - oxo -4, 5- dihydro -1H- pyrazol- 4-ylidine) hydrazinophenyl $\}$ 4-oxoquinazolines "8a- $\mathrm{f}$ ", respectively. Compounds " $\mathrm{a}-\mathrm{f}$ " was established by their correct analytical and spectral data.

Reaction of "5a, 5b" with hydrazine hydrate and methyl hydrazine in refluxing ethanol afforded the corresponding 2-benzyl-3-\{-4-(3-amino-5-oxo-4, 5-dihydro-1Hpyrazol-4-ylidine) hydrazinophenyl -4-oxoquinazolines "9a-d", respectively. The structure of compounds "9a-d" was elucidated from correct analytical and spectral data.

\section{Experimental}

Melting points were determined (by using the open capillary tube method) using a Gallen-Kamp apparatus and are uncorrected. The IR spectra were measured by a unicam SP 1200 spectrophotometer using $\mathrm{KBr}$ discs. The ${ }^{1} \mathrm{H}$ NMR as well as ${ }^{13} \mathrm{C}-\mathrm{NMR}$ were recorded with a Bruker $250 \mathrm{MHz}$ and $62.9 \mathrm{MHz}$, respectively using DMSO- $\mathrm{d}_{6}$ as a solvent and TMS as internal reference, the chemical at $90^{\circ} \mathrm{C}$ inlet temperatures. Elemental analyses were carried out at the CMLS (Cairo University shifts $\delta$ are given in ppm. The mass spectra were run on a 7070 mass spectrometer at $70 \mathrm{eV}$ ). Purity of the compounds was checked on thin layer chromatography (TLC) plates (silica gel G) in the solvent system toluene ethyl formate/formic acid (5:4:1) and benzene-methanol (8:2), the spots were located under iodine vapors or UV light.

Synthesis of-3-(4-aminophenyl)-2-benzyl-4-oxo-3,4-dihydroquinazoline "1a" and 3-(4-aminophenyl)-2-benzyl-6-bromo-4-oxo-3,4-dihydroquinazoline "1b"(18-20)

General procedure

Diazotization of "la, b". Formation of 4- (2- benzyl - 4 - oxo - 3, 4 dihydroquinazolin - 3 - yl) benzene diazonium chloride "2a" and 4-(2-benzyl-6bromo - 4 - oxo - 3, 4-dihydro-quinazolin -3-yl) benzene diazonium chloride "2b":

A well stirred cold solution of "1a" or "1b" $(3.2 \mathrm{~g}, 4.0 \mathrm{~g} ; 0.01 \mathrm{~mol})$ in $3 \mathrm{ml}$ of conc. $\mathrm{HCl}$ and $5 \mathrm{ml}$ of water was diazotized by a cold solution of $\mathrm{NaNO}_{2}(0.7 \mathrm{~g}$; $0.01 \mathrm{~mol}$, dissolved in $1.8 \mathrm{mlof}$ water) stirring was then continued for $30 \mathrm{~min}$, 
then it was triturated with petroleum ether (b.p40-60) to give "2a , $\mathrm{b}$ ", respectively.

Reaction of "2a, b" with acetyl acetone. Formation of 3-\{4-(2-benzyl-4-oxo-3,4dihydroquinazlin-3-yl) phenylhydrazono\}pentane-2,4-diones " $3 a$ " and 3-\{4-(2benzyl-6-bromo-4-oxo-3,4dihydroquinazlin-3-yl)phenyl hydrazonolpentane - 2,4diones " $3 b^{\prime \prime}$

To a cold well stirred solution of acetyl acetone $(1 \mathrm{~g} ; 0.01 \mathrm{~mol})$ in $30 \mathrm{ml}$ of ethanol containing sodium acetate $(4.1 \mathrm{~g} ; 0.05 \mathrm{~mol})$, the diazonium chloride " $2 \mathrm{a}$ " $(3.7 \mathrm{~g}, 0.01 \mathrm{~mol})$ and " $2 \mathrm{~b}$ " $(4.5 \mathrm{~g} ; 0.01 \mathrm{~mol})$ was added, dropwise while stirring during $30 \mathrm{~min}$. Stirring was then continued at room temperature for $30 \mathrm{~min}$ and the solid that separated was filtered off, washed well with dilute ethanol and recrystallized from ethanol to give " $3 \mathrm{a}, \mathrm{b}$ ". (c.f. Table 1).

3a: as yellow crystals IR, " v/cm ${ }^{-1 ": ~} 3422(\mathrm{NH})$; 3058, 2928, $2822(\mathrm{CH}) ; 1717$, 1658(C=O); $1606(\mathrm{C}=\mathrm{N}) ; 1598(\mathrm{C}=\mathrm{C}) .{ }^{1} \mathrm{H}-\mathrm{NMR} ; \delta 11.01$ (s, 1H, NH), 7.31-7.95 $(\mathrm{m}, 13 \mathrm{H}, \mathrm{Ar}-\mathrm{H}), 2.41\left(2 \times \mathrm{s}, 2 \times 3 \mathrm{H}, 2 \times \mathrm{COCH}_{3}\right), 2.12\left(\mathrm{~s}, 2 \mathrm{H}, \mathrm{CH}_{2} \mathrm{pH}\right) .{ }^{13} \mathrm{C}-\mathrm{NMR}$,

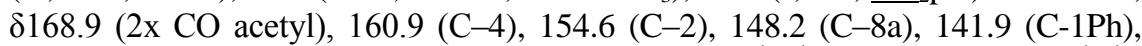
134.9 (C-7), 133.7 (2C Ph, C-2,6), 132.2 (2C Ph, C-1, $\left.4^{\prime}\right), 130.1$ (2CPh, C-2, 6 ), 128.7 (C-5), 127.1 (C-8), 126.4 (2C Ph, C-3,5), 126.1 (C-6), 122.9, (C-4a), 120.9 (2C Ph, C-3', $\left.5^{\downarrow}\right), 119.9(\mathrm{C}-4 \mathrm{Ph}), 29.2\left(\underline{\mathrm{CH}}_{2}-\mathrm{Ph}\right), 23.9\left(2 \mathrm{C}, \mathrm{CH}_{3}\right.$ acetyl). MS, m/z (\%): 439( $\left.\mathrm{M}^{+}+1\right)(19.7), 438\left(\mathrm{M}^{+}\right)(77.9), 395(42.1), 380(67.6), 352(52.2)$ 325(37.4), 225 (33.5), 148(100), 117(3.8), 91(15.1), 77 (11.3), 76 (4.4).

3b: crystallized as yellow needless IR, " v/cm ${ }^{-1 ": 3401}(\mathrm{NH}) ; 3120,2958,2822$ $(\mathrm{CH}), 1707,1669(\mathrm{C}=\mathrm{O}), 1610(\mathrm{C}=\mathrm{N}), 1599(\mathrm{C}=\mathrm{C}) .{ }^{1} \mathrm{H}-\mathrm{NMR}: \delta 11.11(\mathrm{~s}, 1 \mathrm{H}$, $\mathrm{NH}), 7.19-8.01(\mathrm{~m}, 12 \mathrm{H}, \mathrm{ArH}), 2.59\left(2 \mathrm{x} \mathrm{s}, 2 \mathrm{x} 3 \mathrm{H}, 2 \mathrm{xCOCH} \underline{C H}_{3}\right), 2.11(\mathrm{~s}, 2 \mathrm{H}$, $\left.\underline{\mathrm{CH}}_{2} \mathrm{Ph}\right) .{ }^{13} \mathrm{C}-\mathrm{NMR}, \delta 169.3$ (2 x CO acetyl), $163.1(\mathrm{C}-4), 156.1$ (C-2), $149.4(\mathrm{C}-$ 8a), 143.2 (C-1 Ph), 137.3 (C-7), 133.7 (C-4a), 132.6 (C-5), 132.2 (2C Ph, C$\left.1^{\backslash}, 4^{4}\right), 131.9(2 \mathrm{CPh}, \mathrm{C}-2 \backslash, 6), 130.1$ (C-8), 128.7 (2CPh, C-3,5), $128.3(\mathrm{C}-6)$, $128.1\left(2 \mathrm{CPh}, \mathrm{C}-3^{\backslash}, 5^{\backslash}\right), 125.9(\mathrm{C}-4 \mathrm{Ph}), 34.4\left(\underline{\mathrm{CH}}_{2}-\mathrm{Ph}\right), 29.2\left(2 \mathrm{C}, \mathrm{CH}_{3}\right.$ acetyl) MS, $\mathrm{m} / \mathrm{z}(\%) 521\left(\mathrm{M}^{+}+4\right)(31.1), 519\left(\mathrm{M}^{+}+2\right)(30.9), 517\left(\mathrm{M}^{+}\right)$(14.9), 431 (27.6), 404 (76.2), 314 (55.2), 237(31.4), 143(7.6), 90(11.3), 77 (12.9), 76 (14.4).

Reaction of "2a, b" with ethyl acetoacetate. Formation of 2-\{4-(2-benzyl-4-oxo3, 4- dihydroquinazlin-3-yl)phenylhydrazono\}-3-oxobutanoic acid ethyl esters " $4 a, b "$

To a cold, well stirred solution of ethyl acetoacetate $(1.3 \mathrm{~g}, 0.01 \mathrm{~mol})$ in $30 \mathrm{ml}$ of ethanol containing sodium acetate $(4.1 \mathrm{~g}, 0.05 \mathrm{~mol})$ the diazonium chloride derivatives " $2 \mathrm{a}, \mathrm{b}$ " $(3.7 ; 4.5 \mathrm{~g} ; 0.01 \mathrm{~mol})$ was added drop wisely while stirring and the reaction was completed as usual to give "4a, b" ; (c.f. Table 1).

4a: as yellow crystals. IR, " v/cm ${ }^{-1 ": ~} 3343(\mathrm{NH}), 3103,3050,2928(\mathrm{CH}), 1769$, 1701, $1654(\mathrm{C}=\mathrm{O}), 1601(\mathrm{C}=\mathrm{N}), 1589(\mathrm{C}=\mathrm{C}) .{ }^{1} \mathrm{H}-\mathrm{NMR} \delta: 10.59(\mathrm{~s}, 1 \mathrm{H}, \mathrm{NH})$, 7.397 .85 (m, 13H, Ar-H), 3.49 (q, $\left.2 \mathrm{H}, \mathrm{CH}_{2} \mathrm{CH}_{3}\right), 2.45$ (s, 3H, $\left.\mathrm{COCH}_{3}\right), 2.15$ (s, $\left.2 \mathrm{H}, \mathrm{CH}_{2} \mathrm{Ph}\right), 1.39\left(\mathrm{t}, 3 \mathrm{H}, \mathrm{CH}_{2} \underline{\mathrm{CH}}_{3}\right) .{ }^{13} \mathrm{C}-\mathrm{NMR} \delta: 168.6$ (CO acetyl), $164.9(\mathrm{C}=\mathrm{O}$ ester), 161.9 (C-4), 158.1 (C-2), 156.2 (C-8a), 146.8 (C-1 Ph), 141.9 (C-7), Egypt. J. Chem. 54, No. 1 (2011) 
137.2 (C-4a), 133.7 (C-5), 133.1 (2C Ph, C-1 ',4'), 132.2 (2C Ph, C-2',6), 131.9 (C-8), 130.1 (2C Ph, C-3', $\left.5^{\prime}\right), 128.1$ (C-6), 126.3 (2C Ph, C-3', $\left.5^{\prime}\right), 125.9(\mathrm{C}-4$ $\mathrm{Ph}), 29.2\left(\mathrm{CH}_{2}-\mathrm{Ph}\right), 24.1\left(\mathrm{CH}_{3}\right.$ acetyl), $21.3\left(\mathrm{CH}_{3}\right.$ ester $) . \mathrm{MS}, \mathrm{m} / \mathrm{z}(9 \%): 468$ $\left(\mathrm{M}^{+}\right)$(32.4), $423\left(\mathrm{M}^{+}-\mathrm{C}_{2} \mathrm{H}_{5}\right),(19.1), 395\left(\mathrm{M}^{+}-\mathrm{CO}\right)(7.3), 352\left(\mathrm{M}^{+}-\mathrm{COCH}_{3}\right), 219$ (40.1), 118 (85.7), 81(100), 77(50.5).

4b: as yellow crystals. IR, " v/cm ${ }^{-1 "}:(3419(\mathrm{NH}), 3069,3005,2929(\mathrm{CH}), 1774$, 1701, $1660(\mathrm{C}=\mathrm{O}), 1605(\mathrm{C}=\mathrm{N}), 1590(\mathrm{C}=\mathrm{C}) .{ }^{1} \mathrm{H}-\mathrm{NMR} \delta: 11.09(\mathrm{~s}, 1 \mathrm{H}, \mathrm{NH})$, 7.23-8.01 (m, 12H, Ar-H), $4.03\left(\mathrm{q}, 2 \mathrm{H}, \mathrm{CH}_{2} \mathrm{CH}_{3}\right), 2.59$ (s, 3H, $\left.\mathrm{COCH}_{3}\right), 2.19$ (s, $2 \mathrm{H}, \mathrm{CH}_{2} \mathrm{Ph}$ ), 1.40 (t, 3H, $\left.\mathrm{CH}_{2} \underline{\mathrm{CH}}_{3}\right) . \mathrm{MS}, \mathrm{m} / \mathrm{z}$ (40): 548/ 507/546(40) for $\left(\mathrm{M}^{+}\right.$, $\left.{ }^{81} \mathrm{Br},{ }^{80 \mathrm{Br}}{ }^{79} \mathrm{Br}\right), 314$ (49), $298(13.6), 210 / 209 / 208\left(\mathrm{C}_{8} \mathrm{H}_{5} \mathrm{~N}_{2},{ }^{81} \mathrm{Br}, \mathrm{C}_{8} \mathrm{H}_{5} \mathrm{~N}_{2}{ }^{80} \mathrm{Br}\right.$, $\mathrm{C}_{8} \mathrm{H}_{5} \mathrm{~N}_{2}{ }^{79} \mathrm{Br}(85), \quad 158 / 157 / 156\left(\mathrm{C}_{6} \mathrm{H}_{5}{ }^{81} \mathrm{Br}, \quad \mathrm{C}_{6} \mathrm{H}_{5} \quad{ }^{80} \mathrm{Br}, \quad \mathrm{C}_{6} \mathrm{H}_{5}{ }^{79} \mathrm{Br}\right), \quad 81(100)$, 77(55.1).

Reaction of diazonium chloride salts " $2 a, b$ " with ethylcyanoacetate. Formation of: 2-\{-4-(2-benzyl-4-oxo-3, 4- dihydroquinazlin-3-yl)phenylhydrazono\}-3cyanoaceticc acid ethyl esters " $5 a, b$ "

It was prepared as for " $4 \mathrm{a}, \mathrm{b}$ ", using ethyl cyanoacetate as the active methylene compound $(1.1 \mathrm{~g}, 0.01 \mathrm{~mol})$ to give "5a, b" (c.f. Table 1).

5a: as yellow crystals. IR, " v/cm ${ }^{-1 ": ~} 3338(\mathrm{NH}), 3105,3009,2958(\mathrm{CH}), 2242$ $(\mathrm{C} \equiv \mathrm{N}), 1719,1658(\mathrm{C}=\mathrm{O}), 1601(\mathrm{C}=\mathrm{N}), 1598(\mathrm{C}=\mathrm{C}), 1429(\mathrm{~N}=\mathrm{N}-) .{ }^{1} \mathrm{H}-\mathrm{NMR} \delta$ 11.01 (s, 1H, NH), 7.18-7.95 (m, 13H, $\mathrm{Ar}-\mathrm{H}), 3.79$ (q, 2H, $\left.\mathrm{CH}_{2} \mathrm{CH}_{3}\right), 2.20$ (s, $\left.\left.2 \mathrm{H}, \underline{\mathrm{CH}}_{2} \mathrm{Ph}\right), 1.36\left(\mathrm{t}, 3 \mathrm{H}, \mathrm{CH}_{2} \mathrm{CH}_{3}\right) \mathrm{MS}, \mathrm{m} / \mathrm{z}(\%) 452\left(\mathrm{M}^{+}+1\right), 19.4\right), 451\left(\mathrm{M}^{+}\right)$, 78.9), 406 (42.1), 378(31.4), 351 (20.9) 145(100), 102 (4.7), 77(7.1).

5b: as yellow crystals. IR, " v/cm ${ }^{-1 ": ~} 3432(\mathrm{NH}), 3108,3008,2958(\mathrm{CH}), 2245$ $(\mathrm{C} \equiv \mathrm{N}), 1721,1659(\mathrm{C}=\mathrm{O}), 1607(\mathrm{C}=\mathrm{N}), 1589(\mathrm{C}=\mathrm{C}), 1278(-\mathrm{N}-\mathrm{N}=) .{ }^{1} \mathrm{H}-\mathrm{NMR} \delta$ : $10.95(\mathrm{~s}, 1 \mathrm{H}, \mathrm{NH}), 7.09-7.99(\mathrm{~m}, 12 \mathrm{H}, \mathrm{Ar}-\mathrm{H}), 4.01\left(\mathrm{q}, 2 \mathrm{H}, \mathrm{CH}_{2} \mathrm{CH}_{3}\right), 2.57$ (s, $\left.2 \mathrm{H}, \mathrm{CH}_{2} \mathrm{Ph}\right), 1.38\left(\mathrm{t}, 3 \mathrm{H}, \mathrm{CH}_{2} \underline{\mathrm{CH}}_{3}\right) .{ }^{13} \mathrm{C}-\mathrm{NMR} \delta: 164.7(\mathrm{C}=\mathrm{O}$, ester $), 163.2(\mathrm{C}-$ 4), 159.1 (C-2), 143.1 (C-8a), 141.9 (C-1Ph), 137.3 (C-7), 135.2 (2C, C-2, 6 Ph), 133.7, 132.2, 130.1, 128.8 (4C, C-1 $\left.{ }^{`} 2^{\backslash}, 3^{\backslash}, 4^{\backslash}, \mathrm{Ph}\right), 128.1$ (C-4, Ph), $127.9(\mathrm{C}-5)$, $126.3(\mathrm{C}-5 \mathrm{Ph}), 125.9(\mathrm{C}-8), 125.4$ (2C, C-3,5 Ph) 125.51 (C-6), 122.2 (C-4a), $119.5(\mathrm{C} \equiv \mathrm{N}), 59.2\left(\mathrm{CH}_{2}\right), 29.6\left(\mathrm{CH}_{2}-\mathrm{Ph}\right), 15.8\left(\mathrm{CH}_{3}\right.$ ester $) \mathrm{MS}, \mathrm{m} / \mathrm{z}(\%)$ : $532 / 531 / 530 / 529\left(\mathrm{M}^{+}+2, \mathrm{M}^{+}+1, \mathrm{M}^{+}, \mathrm{M}^{+}-1\right)(14.6,14.7,14.5,14.3)$.

Reaction of "3a, $b$ " with guanidine hydrochloride, thiourea and urea, Formation of: 2- benzyl -3- \{4-(2-amino -4, 6- dimethyl\} - 2, 5 -dihydropyrimidin -5ylildine) hydrazinophenyl\} -4-oxoquinazolines "6a,d", 6-bromo-2-benzyl-3-\{4(2-imino-4, 6-dimetyyl-2-thioxopyrimidin-5-ylildine) hydrazinophenyl) - 4 oxoquinazolines "6b-e" and 2- benzyl - 3 - \{4- (2-imino - 4, 6 - dimetyyl - 2 oxopyrimidin - 5 -ylildine) hydrazinophenyl) - 4 - oxoquinazolines " $6 c$, $f^{\prime \prime}$

A mixture of " $3 \mathrm{a}$ " or " $3 \mathrm{~b}$ " $(0.01 \mathrm{~mol})$ and guanidine hydrochloride $(0.59 \mathrm{~g}$, $0.01 \mathrm{~mol})$, thiourea $(076 \mathrm{~g}, 0.01 \mathrm{~mol})$ or urea $(0.6 \mathrm{~g}, 0.01 \mathrm{~mol})$ in $30 \mathrm{ml}$ of absolute ethanol was refluxed for $6 \mathrm{~h}$. After concentration and cooling, the solid that separated was collected, washed well with cold dilute ethanol and recrystallized from the proper solvent as "6a- $\mathrm{f}^{\prime \prime}$ (c.f. Table. 1). 
TABLE 1. Analytical data of the newly prepared compounds.

\begin{tabular}{|c|c|c|c|c|c|c|c|c|}
\hline \multirow{2}{*}{\begin{tabular}{|c} 
Compd. \\
No
\end{tabular}} & \multirow{2}{*}{$\begin{array}{c}\text { Yield } \\
(\%)\end{array}$} & \multirow{2}{*}{$\begin{array}{c}\text { M.P. }{ }^{\circ} \mathbf{C} \\
\text { solvent of } \\
\text { cryst. }\end{array}$} & \multirow{2}{*}{$\begin{array}{c}\text { Molecular } \\
\text { formula } \\
\text { (Mol. w.t) }\end{array}$} & \multicolumn{5}{|c|}{ Elemental analysis cacld/found $(\%)$} \\
\hline & & & & $\mathrm{C}$ & $\mathrm{H}$ & $\mathrm{N}$ & S & $\mathrm{Br}$ \\
\hline 3a & 78 & $\begin{array}{c}158-160 \\
\text { EtOH }\end{array}$ & $\begin{array}{c}\mathrm{C}_{26} \mathrm{H}_{22} \mathrm{~N}_{4} \mathrm{O}_{3} \\
(438)\end{array}$ & $\begin{array}{l}71.23 \\
71.2\end{array}$ & $\begin{array}{l}5.02 \\
5.0\end{array}$ & $\begin{array}{c}12.79 \\
12.8\end{array}$ & & \\
\hline \multirow{2}{*}{$\mathbf{3 b}$} & \multirow{2}{*}{80} & $174-176$ & $\mathrm{C}_{26} \mathrm{H}_{21} \mathrm{~N}_{4} \mathrm{O}_{3} \mathrm{Br}$ & 60.35 & 4.06 & 10.83 & & 15.47 \\
\hline & & $\mathrm{EtOH}$ & (517) & 60.3 & 4.1 & 10.8 & & 15.5 \\
\hline \multirow{2}{*}{$4 a$} & \multirow{2}{*}{72} & 200-202 & $\mathrm{C}_{27} \mathrm{H}_{24} \mathrm{~N}_{4} \mathrm{O}_{4}$ & 69.23 & 5.13 & 11.97 & & \\
\hline & & EtOH & (468) & 69.2 & 5.1 & 12.0 & & \\
\hline \multirow{2}{*}{ 4b } & \multirow{2}{*}{82} & $262-264$ & $\mathrm{C}_{27} \mathrm{H}_{23} \mathrm{~N}_{4} \mathrm{O}_{4} \mathrm{Br}$ & 59.23 & 4.20 & 10.24 & & 14.62 \\
\hline & & $\mathrm{AcOH}$ & $(547)$ & 59.2 & 4.2 & 10.2 & & 14.6 \\
\hline \multirow{2}{*}{$5 \mathbf{a}$} & \multirow{2}{*}{75} & $148-150$ & $\mathrm{C}_{26} \mathrm{H}_{21} \mathrm{~N}_{5} \mathrm{O}_{3}$ & 69.17 & 4.65 & 15.52 & & \\
\hline & & $\mathrm{EtOH}$ & $(451)$ & 69.2 & 4.7 & 15.5 & & \\
\hline \multirow{2}{*}{$5 \mathbf{b}$} & \multirow{2}{*}{81} & $184-186$ & $\mathrm{C}_{26} \mathrm{H}_{20} \mathrm{~N}_{5} \mathrm{O}_{3} \mathrm{Br}$ & 58.86 & 3.77 & 13.207 & & 15.09 \\
\hline & & $\mathrm{AcOH}$ & $(530)$ & 58.9 & 3.8 & 13.2 & & 15.1 \\
\hline \multirow[b]{2}{*}{ 6a } & & 290-291 & $\mathrm{C}_{27} \mathrm{H}_{23} \mathrm{~N}_{7} \mathrm{O}$ & 70.28 & 4.98 & 21.25 & & \\
\hline & 65 & $\mathrm{AcOH}$ & (461) & 70.3 & 5.0 & 21.3 & & \\
\hline $\mathbf{6 h}$ & 62 & $210-212$ & & 67.78 & 4.602 & 17.57 & 6.69 & \\
\hline 6D & 02 & EtOH & $\mathrm{C}_{27} \mathrm{H}_{22} \mathrm{~N}_{6} \mathrm{OS}$ & 67.8 & 4.6 & 17.6 & 6.7 & \\
\hline & 60 & $284-286$ & $\mathrm{C}_{27} \mathrm{H}_{22} \mathrm{~N}_{6} \mathrm{O}_{2}$ & 70.12 & 4.76 & 18.18 & & \\
\hline $\mathbf{~ o c}$ & 00 & $\mathrm{AcOH}$ & $(462)$ & 70.1 & 4.8 & 18.2 & & \\
\hline & & $263-265$ & $\mathrm{C}_{27} \mathrm{H}_{22} \mathrm{~N}_{7} \mathrm{OBr}$ & 60.0 & 4.07 & 18.14 & & 14.81 \\
\hline $\mathbf{6 d}$ & 68 & $\mathrm{AcOH}$ & $(540)$ & 60.0 & 4.1 & 18.1 & & 14.8 \\
\hline & & $217-219$ & $\mathrm{C}_{27} \mathrm{H}_{21} \mathrm{~N}_{6} \mathrm{OSBr}$ & 58.16 & 3.77 & 15.08 & 5.74 & 14.36 \\
\hline $\mathbf{6 e}$ & 61 & $\mathrm{EtOH}$ & (557) & 58.2 & 3.8 & 15.1 & 5.7 & 14.4 \\
\hline & & $234-236$ & $\mathrm{C}_{27} \mathrm{H}_{21} \mathrm{~N}_{6} \mathrm{O}_{2} \mathrm{Br}$ & 59.88 & 3.88 & 15.53 & & 14.78 \\
\hline $6 \mathbf{f t}$ & 13 & $\mathrm{AcOH}$ & $(541)$ & 59.9 & 3.9 & 15.5 & & 14.8 \\
\hline 79 & 60 & $217-219$ & $\mathrm{C}_{32} \mathrm{H}_{26} \mathrm{~N}_{6} \mathrm{O}$ & 75.29 & 5.09 & 16.47 & & \\
\hline$/ \mathrm{a}$ & 00 & $\mathrm{EtOH}$ & (510) & 75.3 & 5.1 & 16.5 & & \\
\hline & & 233-235 & $\mathrm{C}_{32} \mathrm{H}_{25} \mathrm{~N}_{6} \mathrm{OBr}$ & 65.19 & 4.24 & 14.601 & & 13.58 \\
\hline $7 \mathrm{~b}$ & 64 & $\mathrm{AcOH}$ & (589) & 65.2 & 4.2 & 14.6 & & 13.6 \\
\hline $7 c$ & 59 & $263-265$ & $\mathrm{C}_{32} \mathrm{H}_{25} \mathrm{~N}_{7} \mathrm{O}_{3}$ & 69.18 & 4.504 & 17.65 & & \\
\hline$/ \mathrm{c}$ & 59 & $\mathrm{AcOH}$ & (555) & 69.2 & 4.5 & 17.7 & & \\
\hline & & $289-290$ & $\mathrm{C}_{32} \mathrm{H}_{24} \mathrm{~N}_{7} \mathrm{O}_{3} \mathrm{Br}$ & 60.56 & 3.78 & 15.45 & & 12.61 \\
\hline $7 \mathrm{~d}$ & 01 & $\mathrm{AcOH}$ & (634) & 60.6 & 3.8 & 15.5 & & 12.6 \\
\hline $8 \mathbf{a}$ & 50 & $161-163$ & $\mathrm{C}_{25} \mathrm{H}_{20} \mathrm{~N}_{6} \mathrm{O}_{2}$ & 68.807 & 4.58 & 19.27 & & \\
\hline $8 a$ & 50 & $\mathrm{EtOH}$ & (436) & 68.8 & 4.6 & 19.3 & & \\
\hline $8 \mathbf{b}$ & 55 & 213-215 & $\mathrm{C}_{31} \mathrm{H}_{24} \mathrm{~N}_{6} \mathrm{O}_{2}$ & 72.65 & 4.68 & 16.406 & & \\
\hline & & $\mathrm{EtOH}$ & $(512)$ & 72.7 & 4.7 & 16.4 & & \\
\hline $8 c$ & 60 & $265-267$ & $\mathrm{C}_{31} \mathrm{H}_{23} \mathrm{~N}_{7} \mathrm{O}_{4}$ & 66.78 & 4.12 & 17.59 & & \\
\hline $\mathbf{~ o c}$ & 00 & $\mathrm{AcOH}$ & $(557)$ & 66.8 & 4.1 & 17.6 & & \\
\hline $8 d$ & 53 & $257-259$ & $\mathrm{C}_{25} \mathrm{H}_{19} \mathrm{~N}_{6} \mathrm{O}_{2} \mathrm{Br}$ & 58.25 & 3.63 & 16.31 & & 15.53 \\
\hline $8 \mathrm{a}$ & 53 & $\mathrm{AcOH}$ & $(515)$ & 58.3 & 3.7 & 16.3 & & 15.5 \\
\hline & & 273-275 & $\mathrm{C}_{31} \mathrm{H}_{23} \mathrm{~N}_{6} \mathrm{O}_{2} \mathrm{Br}$ & 62.94 & 3.89 & 14.21 & & 13.53 \\
\hline $8 \mathrm{e}$ & 60 & $\mathrm{EtOH}$ & (591) & 62.9 & 3.9 & 14.2 & & 13.5 \\
\hline $8 f$ & 65 & $263-265$ & $\mathrm{C}_{31} \mathrm{H}_{22} \mathrm{~N}_{7} \mathrm{O}_{4} \mathrm{Br}$ & 58.49 & 3.45 & 15.408 & & 12.57 \\
\hline $8 \mathrm{I}$ & 65 & $\mathrm{AcOH}$ & (636) & 58.5 & 3.5 & 15.4 & & 12.6 \\
\hline 0 & 60 & $179-181$ & $\mathrm{C}_{24} \mathrm{H}_{19} \mathrm{~N}_{7} \mathrm{O}_{2}$ & 65.90 & 4.35 & 22.43 & & \\
\hline $9 a$ & 60 & $\mathrm{EtOH}$ & $(437)$ & 65.9 & 4.3 & 22.4 & & \\
\hline $9 \mathbf{b}$ & 65 & $243-245$ & $\mathrm{C}_{24} \mathrm{H}_{18} \mathrm{~N}_{7} \mathrm{O}_{2} \mathrm{Br}$ & 55.81 & 3.48 & 18.99 & & 15.503 \\
\hline & 65 & $\mathrm{AcOH}$ & (516) & 55.8 & 3.5 & 19.0 & & 15.5 \\
\hline $9 c$ & 63 & $187-189$ & $\mathrm{C}_{25} \mathrm{H}_{21} \mathrm{~N}_{7} \mathrm{O}_{2}$ & 66.51 & 4.65 & 21.72 & & \\
\hline $9 \mathrm{c}$ & 03 & $\mathrm{EtOH}$ & $(451)$ & 66.5 & 4.7 & 21.7 & & \\
\hline & & $269-271$ & $\mathrm{C}_{25} \mathrm{H}_{20} \mathrm{~N}_{7} \mathrm{O}_{2} \mathrm{Br}$ & 56.603 & 3.77 & 18.49 & & 15.09 \\
\hline $9 d$ & 65 & $\mathrm{AcOH}$ & $(530)$ & 56.6 & 3.8 & 18.5 & & 15.1 \\
\hline
\end{tabular}

where $\mathrm{EtoH}=$ ethanol and $\mathrm{AcoH}=$ Acetic acid. 
6a: as yellow crystals. IR, " v/cm ${ }^{-1 ":} 3420(\mathrm{NH}), 3058,2982(\mathrm{CH}), 1671(\mathrm{CO})$, $1614(\mathrm{C}=\mathrm{N}), 1601(\mathrm{C}=\mathrm{C}) .{ }^{1} \mathrm{H}-\mathrm{NMR} \delta: 11.01(\mathrm{~s}, 1 \mathrm{H}, \mathrm{NH}-\mathrm{Ph}), 10.91(\mathrm{~s}, 1 \mathrm{H}$, $\mathrm{C}=\mathrm{NH}), 7.01-7.95$ (m, $13 \mathrm{H}, \mathrm{Ar}-\mathrm{H}), 3.14$ (s, 2H, $\left.\mathrm{CH}_{2} \mathrm{pH}\right), 2.13$ (s, 6H, 2x CH$)_{3}$. ${ }^{13} \mathrm{C}-\mathrm{NMR} \delta$ : 162.7 (C-4 quinazolinone), 155.2 (C-2, quinazolinone), 153.1 (C2, pyrimidine), 152.8 (C-6, pyrimidine), 151.9 (C-4, pyrimidine), 150.2 (C-5, pyrimidine), 149.8 (C-8a), 148.1 (C-1, Ph), 147.3 (C-4, Ph), 145.2 (C-7), 142.7 (2C, C-2,6 Ph), 140.7 (C-8), 138.1 (C-5), 133.1, 132.8, 130.9, 129.3 (4C, C$\left.1^{\prime}, 2^{\backslash}, 3^{\backslash}, \mathrm{Ph}\right), 128.6(\mathrm{C}-4, \mathrm{Ph}), 128.1(\mathrm{C}-8), 127.4(\mathrm{C}-5), 126.6(2 \mathrm{C} \mathrm{C}-3,5 \mathrm{Ph})$, 126.3 (C-6), 125.9 (C-4a), 126.3 (4C, C-2 $\left.3^{\backslash}, 5^{\prime}, 6^{\prime}\right), 125.9$ (C-4 Ph), $122.2\left(\mathrm{C}-1^{\prime}\right.$ $\mathrm{Ph}), 26.9\left(\mathrm{CH}_{2}-\mathrm{Ph}\right), 21.3\left(2 \mathrm{C}, 2 \mathrm{x}\right.$ pyrimidine $\left.\mathrm{CH}_{3}\right)$.

6b: as dark yellow crystals, IR, " v/ $\mathrm{cm}^{-1 ": ~ 3437(N H), ~ 3093, ~ 2921, ~} 2852(\mathrm{CH})$, $1673(\mathrm{C}=\mathrm{O}), 1608(\mathrm{C}=\mathrm{N}), 1598(\mathrm{C}=\mathrm{C}), 1279(-\mathrm{N}-\mathrm{N}=), 1218(\mathrm{C}=\mathrm{S}) .{ }^{1} \mathrm{H}-\mathrm{NMR} \delta$ : 11.09 (s, 1H, NH-Ph), 7.18-8.31 (m, 13H, $\mathrm{Ar}-\mathrm{H}), 2.95$ (s, $\left.2 \mathrm{H}, \mathrm{CH}_{2} \mathrm{Ph}\right), 2.11$ (2xs, 6H, 2xCH $)$. MS, m/z (\%): $479\left(\mathrm{M}^{+}+1\right)(14.6), 478\left(\mathrm{M}^{+}\right),(14.6), 348$ (20.9), 243 (23.9), 152 (100), 145 (67.1), 105 (16.1), 92 (12.3), 77(7.5), 76 (4.9).

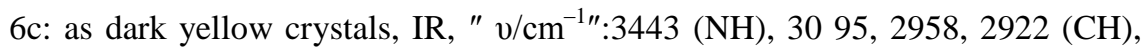
1679, $1668(\mathrm{C}=\mathrm{O}), 1601,(\mathrm{C}=\mathrm{N}), 1595(\mathrm{C}=\mathrm{C}), 1425(-\mathrm{I}-\mathrm{l}-) .{ }^{1} \mathrm{H}-\mathrm{NMR} \delta$ 11.4 (s, 1H, NH-Ph) , 7.09-8.19 (m, 13H, Ar-H), 3.14 (s, 2H, $\left.\mathrm{CH}_{2} \mathrm{Ph}\right), 2.25$ (s, $\left.6 \mathrm{H}, 2 \times \mathrm{CH}_{3}\right) .{ }^{13} \mathrm{C}-\mathrm{NMR} \quad \delta: 168.2(\mathrm{C}-2$, pyrimidinone), $166.8(\mathrm{C}-4$, quinazolinone), 163.8 (C-6, pyrimidinone), 162.4 (C-4, pyrimidinone),157.1 (C5, pyrimidin-2-one), 155.9 (C-2, quinazolinone), 149.8 (C-8a), 145.3 (C-1, Ph), 136.1 (C-7), 133.5 (2C, C-2, $6 \mathrm{Ph}), 132.6$ (2C, C-3,5 Ph), 131.9 (C-4Ph) 130.1 (C-8), 128.3 (C-5), 128.1 (C-6), 126.6 (C-4a), 126.3 (C-1'), 125.9 (2C, C$\left.2^{`}, 6 \mathrm{Ph}\right), 125.4\left(2 \mathrm{C}, \mathrm{C}-3{ }^{\prime}, 5^{`} \mathrm{Ph}\right), 125.1\left(\mathrm{C}-4^{\prime}\right), 29.3\left(\underline{\mathrm{CH}}_{2} . \mathrm{Ph}\right), 21.4(2 \mathrm{C}, 2 \mathrm{x}$ pyrimidin-2-one $-\mathrm{CH}_{3}$ ).

6d: as yellowish orange crystals. IR, " v/cm ${ }^{-1 "}: 3444(\mathrm{NH}), 3090,2982,2858$ $(\mathrm{CH}), 1678(\mathrm{C}=\mathrm{O}), 1607(\mathrm{C}=\mathrm{N}), 1595(\mathrm{C}=\mathrm{C}), 1278(-\mathrm{N}-\mathrm{N}=) .{ }^{1} \mathrm{H}-\mathrm{NMR} \delta: 10.99$ (s, $1 \mathrm{H}, \mathrm{NH}, \mathrm{Ph}), 7.12-8.23(\mathrm{~m}, 12 \mathrm{H}, \mathrm{Ar}-\mathrm{H}), 3.18\left(\mathrm{~s}, 2 \mathrm{H}, \mathrm{CH}_{2} \mathrm{Ph}\right), 2.53$ ( 2 x s, $\left.6 \mathrm{H}, 2 \mathrm{x} \mathrm{CH}_{3}\right) . \mathrm{MS}, \mathrm{m} / \mathrm{z}(\%): 542\left(\mathrm{M}^{+}+2\right)(17.6), 540\left(\mathrm{M}^{+}\right)(17.8), 539\left(\mathrm{M}^{+}-1\right)$ (17.6), 314 (19.6), 234 (12.7), 218 (37.9), 150 (67.2), 134(100), 92 (37.1), 77(4.9), 76(3.8).

6e: as light orange crystals. IR, " v/ $\mathrm{cm}^{-1 "}: 3358(\mathrm{NH}), 3093,2928,2852(\mathrm{CH})$, $1671(\mathrm{C}=\mathrm{O}), 1605(\mathrm{C}=\mathrm{N}), 1598(\mathrm{C}=\mathrm{C}), 1258(\mathrm{C}=\mathrm{S}) .{ }^{1} \mathrm{H}-\mathrm{NMR} \delta: 10.95(\mathrm{~s}, 1 \mathrm{H}$, NH.Ph) 7.19-8.30 (m, 12H, Ar-H), $3.21\left(\mathrm{~s}, 2 \mathrm{H}, \mathrm{CH}_{2} \mathrm{Ph}\right), 2.59\left(2 \times 5,6 \mathrm{H}, 2 \mathrm{x} \mathrm{CH}_{3}\right)$. ${ }^{13} \mathrm{C}$-NMR $\delta: 162.7$ (C-4, quinazolinone), 159.5 (C-2, Pyrimidine-2-thione), 157.4 (C-5, pyrimidine-2-thione), 155.9 (C-6, pyrimidin-2-thione), 155.3 (C-2, quinazolinone), 150.3 (C-8a), 145.6 (C-1, Ph), 136.3 (C-7), 133.3 (2C, C-3,6 $\mathrm{Ph}), 132.2$ (2C, C3,5 Ph), 131.9 (C-4 Ph), 130.1 (C-8), 128.6 (C-5), 128.1 (C-6), 126.3 (C-4a), $125.9\left(\mathrm{C}^{\prime} 1^{\prime}\right), 125.4$ (2C, C-2',6 Ph), 122.2 (2C, 3', $\left.5^{\prime} \mathrm{Ph}\right), 121.7$ (C$4 \mathrm{Ph}), 29.1\left(\mathrm{CH}_{2} \cdot \mathrm{Ph}\right), 21.3\left(2 \mathrm{C}, 2 \mathrm{x}\right.$ pyrimidin-2-thione $\left.\mathrm{CH}_{3}\right)$. 


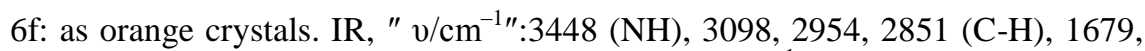
$1666(\mathrm{C}=\mathrm{O}), 1608(\mathrm{C}=\mathrm{N}), 1578(\mathrm{C}=\mathrm{C}), 1262(\mathrm{~N}-\mathrm{N}=) .{ }^{1} \mathrm{H}-\mathrm{NMR} \delta: 11.01(\mathrm{~s}, 1 \mathrm{H}$, $\mathrm{NH}), 7.23-8.01(\mathrm{~m}, 12 \mathrm{H}, \mathrm{Ar}-\mathrm{H}), 2.45\left(2 \mathrm{x} \mathrm{s}, 6 \mathrm{H}, 2 \mathrm{x} \mathrm{CH}_{3}\right.$ pyrimidin-2-one). MS, m/z (\%): $543\left(\mathrm{M}^{+}+2\right),(19.6), 542\left(\mathrm{M}^{+}+1\right)(19.6), 541\left(\mathrm{M}^{+}\right)(19.6), 316,314$ (26.1). 227, 225 (47), 170 (18), 151 (66), 89(38), 76(23), 75(13), 62(100).

Reaction of "3a, $b$ " with hydrazines. Formation of 2-benzyl-3-\{4-(3,5-dimethyl-1phenyl-1-H-pyrazol-4-yl) azophenyl\}- 4-oxoquinazolines "7a", 2-benzyl-6-bromo3-\{4-(3,5-dimethyl-1-phenyl-1H-pyrazol-4-yl)-4-oxoquinazolines "7b", 2-benzyl-314-(3,5- dimethyl-1(-4-nitrophenyl-1H-pyrazol-4-yl)-4 oxoquinazolines "7c" and 2- benzy l- 6- bromo -3- 44-(3,5-dimethyl-1(-4-nitrophenyl-1H-pyrazol-4-yl)-4oxoquinazolines " $7 d^{\prime \prime}$

A mixture of "3a,b" (0.01 mol), phenyl hydrazine (1g, $0.01 \mathrm{~mol})$, 4nitrophenyl hydrazine $(1.5 \mathrm{~g}, 0.01 \mathrm{~mol})$ and freshly fused sodium acetate $(0.8 \mathrm{~g}$, $0.01 \mathrm{~mol}$ ) in $30 \mathrm{ml}$ of acetic acid was refluxed for 6hrs. After cooling, the excess solvent was evaporated (under pressure), and the product that separated was collected, washed with ether, then with dilute alcohol and recrystallized from the proper solvent to give "7a-d" (c.f. Table 1).

7a: as yellow orange crystals. IR, "v/ $\mathrm{cm}^{-1 "}: 3064,3016,2954,2921,2851(\mathrm{CH})$, 1667(C=O,quinazolinone), $1608(\mathrm{C}=\mathrm{N}), 1424(-\mathrm{N}=\mathrm{N}-) .{ }^{1} \mathrm{H}-\mathrm{NMR} \quad \delta: 7.73-7.98$ (m, 18H, Ar-H), 2.82-2.83 (2x s, 2 x 3H, 2x CH -pyrazole ring, 2.35 (s, $2 \mathrm{H}, \underline{\mathrm{CH}}_{2}$. $\mathrm{Ph}$ ). ${ }^{13} \mathrm{C}$-NMR $\delta$ : 163.7 (C-4 quinazolinone), 159.5 (C-2, quinazolinone, 154.7 (C-8a quinazolinone), 153.9 (C-4a quinazolinone, 153.1 (C-4 pyrazole), 144.8 (2C, C-1,4 Ph), 142.7 (2C, C-3,5 pyrazole), 133.6 (2C, C-2,6 Ph), 132.6 (2C, C$3,5 \mathrm{Ph}), 132.2$ (Cl-phenyl pyrazole), 130.1 (2C, C-2,6 phenyl pyrazole), 128.3 (C-4 phenyl pyrazole), 126.6 (2C, C-3,5 phenyl pyrazole), $126.3\left(\mathrm{C}^{1}{ }^{\prime} \mathrm{Ph}\right), 125.9$ $\left(\mathrm{C}-4^{\backslash} \mathrm{Ph}\right), 125.4\left(2 \mathrm{C}, \mathrm{C}-2^{\prime}, 6^{\prime} \mathrm{Ph}\right), 122.2\left(2 \mathrm{C}, \mathrm{C}-3^{\backslash}, 5^{\backslash} \mathrm{Ph}\right), 29.6\left(\underline{\mathrm{CH}}_{2}-\mathrm{Ph}\right), 21.6$ (2C, $2 \mathrm{x} \mathrm{CH}_{3}$ pyrazole).

7b: as dark orange crystals. IR, " v/cm ${ }^{-1 ": 3103, ~ 3090, ~ 2984, ~ 2921, ~} 2823(\mathrm{CH})$, $1671\left(\mathrm{C}=\mathrm{O}\right.$, quinazolinone), 1609, $1598(\mathrm{C}=\mathrm{N}$ and $\mathrm{C}=\mathrm{C}), 1428(-\mathrm{N}=\mathrm{N}-) .{ }^{1} \mathrm{H}-$ NMR $\delta$ : 7.79-8.09 (m, 17H, Ar-H), 2.91, $2.95\left(2 \times s, 2 \times 3 \mathrm{H}, 2 \mathrm{C} \mathrm{CH}_{3}\right.$-pyrazole ring), 2.49 (s, $\left.2 \mathrm{H}, \mathrm{CH}_{2}-\mathrm{Ph}\right)$. MS, m/z(\%): $593\left(\mathrm{M}^{+}+4\right)(4.5), 591\left(\mathrm{M}^{+}+2\right)(4.5)$, $589\left(\mathrm{M}^{+}\right)(27.7), 418(11.1), 224(37.1) 171$ (3.5), 199 (19.1), 144 (15.7), 129 (23), 96 (11.1), 77(23.3), 76(9.1), 55(100).

7c: as reddish crystals. IR, " v/cm ${ }^{-1 ": 3080, ~ 3005, ~ 2955, ~ 2922, ~} 2825(\mathrm{CH}), 1676$ $\left(\mathrm{C}=\mathrm{O}\right.$, quinazoline), $1601(\mathrm{C}=\mathrm{N}), 1588(\mathrm{C}=\mathrm{C}), 1523(\mathrm{~N}=\mathrm{O}), 1419(-\mathrm{N}=\mathrm{N}) .{ }^{1} \mathrm{H}-$ NMR $\delta: 7.46-8.13(\mathrm{~m}, 17 \mathrm{H}, \mathrm{Ar}-\mathrm{H}), 2.61,2.54\left(2 \mathrm{x} \mathrm{s}, 2\right.$ x 3H, 2 x $\mathrm{CH}_{3}$-pyrazole ring), 2.51 (s, $\left.2 \mathrm{H}, \underline{\mathrm{CH}}_{2} . \mathrm{Ph}\right)$.

7d: as dark red crystals. IR, " v/cm ${ }^{-1 ": 3103, ~ 3080, ~ 2958, ~ 2921, ~} 2852(\mathrm{CH}), 1674$ $(\mathrm{C}=\mathrm{O}$, quinazolinone), $1605(\mathrm{C}=\mathrm{N}), 1591(\mathrm{C}=\mathrm{C}), 1523(\mathrm{~N}=\mathrm{O}), 1414(-\mathrm{N}=\mathrm{N}-)$. MS, $\mathrm{m} / \mathrm{z}(\%): 635\left(\mathrm{M}^{+}+1\right)(16.7), 634\left(\mathrm{M}^{+}\right)(19.2), 633\left(\mathrm{M}^{+}-1\right)(19.2), 554(7.2)$, 320 (33.6), 215(17.1), 122(9.3), 96(100).

Egypt. J. Chem. 54, No. 1 (2011) 
Reaction of " $4 a, b$ " with hydrazines. Formation of: 2-benzyl-3-\{4-(3-methyl-5-oxo4, 5-dihydro-1H-pyrazol-4-ylidine)hydrazinophenyl $\}$-4-oxoquinazolines "8a-f"

A mixture of "4a ,b" (0.01 mol), and hydrazine hydrate $(0.5 \mathrm{~g}, 0.01 \mathrm{~mol})$,or 4 nitrophenylhydrazine $(1.0 \mathrm{~g}, 0.01 \mathrm{~mol})$ in $15 \mathrm{ml}$ of acetic acid containing sodium acetate $(0.41 \mathrm{~g}, 0.005 \mathrm{~mol})$ was refluxed for $6 \mathrm{hr}$. After concentration and cooling, the excess solvent was removed (reduced pressure) and the product was collected washed well with dilute alcohol and recrystallized from the proper solvent to give "8a-f" ( $c f$. Table 1).

8a: crystallized as yellow crystals. IR, " v/cm ${ }^{-1 "}: 3466,3324(\mathrm{NH}), 3030,3005$, $2958(\mathrm{CH}), 1665,1672(\mathrm{C}=\mathrm{O}), 1601(\mathrm{C}=\mathrm{N}), 1589(\mathrm{C}=\mathrm{C}) .{ }^{1} \mathrm{H}-\mathrm{NMR} \delta: 11.11(\mathrm{~s}$, 1H, Ph. NH.N=), 10.09 (s, 1H, NH-pyrazolone), 7.23-8.01 (m, 13H, Ar-H), 2.49 (s, $\left.2 \mathrm{H}, \mathrm{CH}_{2} \mathrm{Ph}\right), 2.13$ (s, 3H, $\mathrm{CH}_{3}$-pyrazolone). ${ }^{13} \mathrm{C}-\mathrm{NMR} \quad \delta: 159.1$ (C-4 quinazolinone), 158.4 (C-5 pyrazolone), 156.1 (C-4 pyrazolone), 155.2 (C-2 quinazolone), 152.4 (C-3 pyrazolone), 149.7 (C-8a), 148.1 (C-1, Ph), 147.3 (C-4 $\mathrm{Ph}), 145.3$ (C-7), 149.7 (C-8a), 148.1 (C-1, Ph), 147.3 (C-4 Ph), 145.3 (C-7), 142.6 (2C, C-2,6 Ph), 140.5 (C-8), 138.2 (C-5), 133.7, 132.6, 132.2, 130.1 (4C, C-1', 2', 3', $\left.4^{`} \mathrm{Ph}\right), 128.1$ (C-8), 126.3 (2C, C-3,5 Ph), 125.9 (C-6), 125.4 (C-4a), $122.2\left(2 \mathrm{C}, 5^{\prime}, 6^{\prime}\right), 24.1\left(\mathrm{CH}_{2} \cdot \mathrm{Ph}\right), 23.9\left(\mathrm{CH}_{3}\right.$, pyrazolone $)$.

8b: light orange crystals. IR, " v/cm ${ }^{-11 "}: 3442(\mathrm{NH}), 3130,3005,2928,2828(\mathrm{C}-$ H), 1664, $1679(\mathrm{C}=\mathrm{O}), 1605,1599$ (C=N, C=C). ${ }^{1} \mathrm{H}-\mathrm{NMR} \delta: 11.12$ (s, 1H, NHN-Ph) 7.21-8.09 (m, 18H, Ar-H), $2.48\left(\mathrm{~s}, 2 \mathrm{H}, \mathrm{CH}_{2} \mathrm{Ph}\right) 2.135\left(\mathrm{~s}, 3 \mathrm{H}, \mathrm{CH}_{3}-\right.$ pyrazolinone). MS, $\mathrm{m} / \mathrm{z}(\%), 513\left(\mathrm{M}^{+}+1,73.2\right), 512\left(\mathrm{M}^{+}, 41.5\right)$.

8c: dark orange crystals. IR, " v/cm $\mathrm{cm}^{-1 ":} 3440.9(\mathrm{NH}), 3090,3005,2982,2928$, $2828(\mathrm{C}-\mathrm{H}), 1669,1678(\mathrm{C}=\mathrm{O}), 1604,1598(\mathrm{C}=\mathrm{N}, \mathrm{C}=\mathrm{C}) .{ }^{1} \mathrm{H}-\mathrm{NMR} \delta: 11.39$ (s, $1 \mathrm{H}, \underline{\mathrm{NH}}-\mathrm{N}-\mathrm{Ph}), 7.38-8.13(\mathrm{~m}, 23 \mathrm{H}, \mathrm{Ar}-\mathrm{H}), 2.51\left(\mathrm{~s}, 2 \mathrm{H}, \mathrm{CH}_{2} \mathrm{Ph}\right), 2.35(\mathrm{~s}, 3 \mathrm{H}$, $\mathrm{CH}_{3}-$ pyrazolinone). $\mathrm{MS}, \mathrm{m} / \mathrm{z}(\%): 558\left(\mathrm{M}^{+}+1,78.9\right), 557\left(\mathrm{M}^{+}, 52.1\right) 232(37.6)$, 205(100), 109 (87.1), 84(37.4), 76 (71.13), 54(57.3).

8d: yellowish orange crystals. IR, " v/cm ${ }^{-1 ":} 3451.1(\mathrm{~N} \mathrm{H}), 3110,3030,3005$, 2928, $2852(\mathrm{C}-\mathrm{H})$, 1668, $1674(\mathrm{C}=\mathrm{O}), 1601,1599(\mathrm{C}=\mathrm{N}, \mathrm{C}=\mathrm{C}) .{ }^{1} \mathrm{H}-\mathrm{NMR} \delta: 11.31$ (s, $1 \mathrm{H}, \underline{\mathrm{NH}}-\mathrm{N}-\mathrm{Ph}), 7.39-8.35(\mathrm{~m}, 19 \mathrm{H}, \mathrm{Ar}-\mathrm{H}), 2.601$ (s, $\left.2 \mathrm{H}, \mathrm{CH}_{2} \mathrm{Ph}\right), 2.36$ (s, 3H, $\mathrm{CH}_{3}$-pyrazolone). $\mathrm{MS}, \mathrm{m} / \mathrm{z}(\%): 516\left(\mathrm{M}^{+} 1\right.$ for $\left.{ }^{81} \mathrm{Br}, 19.37\right), 515\left(\mathrm{M}^{+}\right.$for $\left.{ }^{80} \mathrm{Br}, 19.37\right)$ $514\left(\mathrm{M}^{+}\right.$for $\left.{ }^{79} \mathrm{Br}, 63.1\right), 146\left(\mathrm{C}_{8} \mathrm{H}_{6} \mathrm{~N}_{2} \mathrm{O}^{+}, 73.1\right),\left(\mathrm{C}_{6} \mathrm{H}_{6} \mathrm{~N}^{+}, 100\right) 52(76)$.

8e: Dark orange crystals. IR, " v/cm ${ }^{-1 ": ~} 3450(\mathrm{NH}), 3151,3030,3008,2982$, 2928, $2822(\mathrm{C}-\mathrm{H}), 1669,1680(\mathrm{C}=\mathrm{O}), 1605,1603,1590(\mathrm{vC}=\mathrm{N}), \mathrm{C}=\mathrm{C}) .{ }^{1} \mathrm{H}-$ NMR $\delta$ : $11.321(\mathrm{~s}, 1 \mathrm{H}, \underline{\mathrm{NH}}-\mathrm{NPh}), 7.41-8.34(\mathrm{~m}, 23 \mathrm{H}$, Ar-H), $2.61(\mathrm{~s}, 2 \mathrm{H}$, $\left.\mathrm{CH}_{2} \mathrm{Ph}\right), 2.51$ (s, 3H, $\mathrm{CH}_{3}$-pyrazolinone), MS, m/z (\%) $592\left(\mathrm{M}^{+}+1\right.$, for ${ }^{81} \mathrm{Br}$, 19.2), $591\left(\mathrm{M}^{+}\right.$for $\left.{ }^{80} \mathrm{Br}, 20.1\right), 590\left(\mathrm{M}^{+}-1\right.$ for $\left.{ }^{79} \mathrm{Br}, 20.1\right)$

8f: Reddish crystals. IR, " v/cm ${ }^{-1 ":} 455(\mathrm{NH}), 3051,3030,3009,2982,2928$, $2828(\mathrm{C}-\mathrm{H}), 1673,1680(\mathrm{C}=\mathrm{O}), 1605,1601,1590(\mathrm{C}=\mathrm{N}, \mathrm{C}=\mathrm{C}) .{ }^{1} \mathrm{H}-\mathrm{NMR} \delta: 11.68$ (s, 1H, NH-N-Ph), 7.41-8.26 (m, 16H, Ar-H), $2.62\left(\mathrm{~s}, 2 \mathrm{H}, \mathrm{CH}_{2} \mathrm{Ph}\right), 2.49$ (s, 3H, $\mathrm{CH}_{3}$-pyrazolinone). MS, $\mathrm{m} / \mathrm{z}(\%): 627\left(\mathrm{M}^{+}+1\right.$ for $\left.{ }^{81} \mathrm{Br}, 11,2\right), 636\left(\mathrm{M}^{+}\right.$for ${ }^{80} \mathrm{Br}$, 70.9), $635\left(\mathrm{M}^{+}-1{ }^{79} \mathrm{Br}, 71.1\right)$. 
Reaction of "5a, $b$ " with hydrazines. Formation of: 2-benzyl-3-\{-4-(3-amino-5-oxo4, 5-dihydro-1Hpyrazol-4-ylidine) hydrazinophenyl\}-4-oxoquinazolines "9a-d"

A mixture of " $5 \mathrm{a}, \mathrm{b}$ " $(0.01 \mathrm{~mol})$ and hydrazines namely hydrazine hydrate or methyl hydrazine $(1.0 \mathrm{gm}, 0.01 \mathrm{~mol})$ in $30 \mathrm{ml}$ of ethanol was refluxed for $6 \mathrm{hr}$. After concentration and cooling, the excess of solvent was removed and the product was collected and recrystalized from the proper solvent to give "9a-d" (c.f. Table 1).

9a: yellowish orange crystals. IR, " v/cm ${ }^{-11 ": ~ 430, ~ 3452(N H), ~ 3310(~}\left(\mathrm{NH}_{2}\right), 3156$, $2928(\mathrm{CH}), 1679,1669(\mathrm{C}=\mathrm{O}), 1605,1589,1509(\mathrm{C}=\mathrm{N}, \mathrm{C}=\mathrm{C}) .{ }^{1} \mathrm{H}-\mathrm{NMR} \quad \delta$ : 11.67(s, $\left.{ }^{1} \mathrm{HNH}-\mathrm{N}=\right), 10.01$ (s, 1H, NH-pyrazolinone), 7.41-8.13(m, 13H, Ar-H), 5.05 (s, $2 \mathrm{H}, \underline{\mathrm{NH}}_{2}-$ pyrazolinone), 2.61 (s, $\left.2 \mathrm{H}, \underline{\mathrm{CH}}_{2}-\mathrm{Ph}\right) . \mathrm{MS}, \mathrm{m} / \mathrm{z}(\%), 438\left(\mathrm{M}^{+}+1\right)$ (14.3), $437\left(\mathrm{M}^{+}\right)$(37.25), $327\left(\mathrm{C}_{21} \mathrm{H}=17 \mathrm{O}^{+} \mathrm{N}_{3}\right)(71.1), 312\left(\mathrm{C}_{21} \mathrm{H}_{16} \mathrm{O}^{+} \mathrm{N}_{2}\right), 236$ $\left(\mathrm{C}_{15} \mathrm{H}_{12} \mathrm{O}^{+} \mathrm{N}_{2}\right) \quad(100), 160\left(\mathrm{C}_{9} \mathrm{H}_{8} \mathrm{O}^{+} \mathrm{N}_{2}\right) \quad(41.1), \quad 146\left(\mathrm{C}_{8} \mathrm{H}_{6} \mathrm{O}^{+} \mathrm{N}_{2}\right) \quad(27.5), 104$ $\left(\mathrm{C}_{7} \mathrm{H}_{4} \mathrm{O}^{+}\right)(2.7), 77(83.3), 76(18.3), 55(71.4)$.

9b: Orange crystals. IR, " v/cm ${ }^{-1 "}: 434,3451(\mathrm{NH}), 3316\left(\mathrm{NH}_{2}\right), 3159$, 2952,2928, 2882, 2826 (VH), 1678, $1666(\mathrm{C}=\mathrm{O}), 1604,1601,1561(\mathrm{C}=\mathrm{N}, \mathrm{C}=\mathrm{C})$ ${ }^{1} \mathrm{H}-\mathrm{NMR} \quad \delta: 11.69$ (s, $\left.{ }^{1} \mathrm{H}-\mathrm{NM}-\mathrm{N}=\right), 10.12$ (s, 1H, NH-pyrazolinone), 7.52-8.21 (m, 12H, Ar-H), 5.13 (s, 2H, $\underline{\mathrm{NH}}_{2}$-Pyrazolinone), $2.63\left(\mathrm{~s}, 2 \mathrm{H}, \underline{\mathrm{CH}_{2}} \mathrm{Ph}\right)$.

9c: golden yellow crystals. IR, " v/cm ${ }^{-1 "}: 3438(\mathrm{NH}), 3312\left(\mathrm{NH}_{2}\right), 3030,2928$, 2882, 2828(CH), 1674, $1666\left(\mathrm{C}_{2} \mathrm{O}\right), 1605,1601,1588(\mathrm{C}=\mathrm{N}, \mathrm{C}=\mathrm{C}) .{ }^{1} \mathrm{H}-\mathrm{NMR} \delta$ : $11.59(\mathrm{~s}, 1 \mathrm{H}, \mathrm{NH}-\mathrm{N}=), 7.43-8.21(\mathrm{~m}, 13 \mathrm{H}, \mathrm{Ar}-\mathrm{H}), 5.11\left(\mathrm{~s}, 2 \mathrm{H}, \mathrm{NH}_{2}\right.$ pyrazolinone), 2.39 (s, $\left.2 \mathrm{H}, \underline{\mathrm{CH}}_{2} \cdot \mathrm{Ph}\right), 1.31\left(\mathrm{~s}, 3 \mathrm{H}, \underline{\mathrm{CH}}_{3}-\mathrm{N}\right)$

9d: light orange crystals. IR, " v/cm ${ }^{-1 "}: 3438(\mathrm{NH}), 3321\left(\mathrm{NH}_{2}\right), 3150,3050$, 3003, 2928, $2828(\mathrm{CH}), 1679,1669(\mathrm{C}=\mathrm{O}), 1606,1602,1580(\mathrm{C}=\mathrm{N}, \mathrm{C}=\mathrm{C}) .{ }^{1} \mathrm{H}-$ NMR $\delta: 11.61(\mathrm{~s}, 1 \mathrm{H}, \underline{\mathrm{NH}}-\mathrm{N}=), 7.45-8.31(\mathrm{~m}, 12 \mathrm{H}, \mathrm{Ar}-\mathrm{H}), 5.14\left(\mathrm{~s}, 2 \mathrm{H}, \mathrm{NH}_{2}-\right.$ pyrazolinone), $2.63\left(\mathrm{~s}, 2 \mathrm{H}, \mathrm{CH}_{2} \mathrm{Ph}\right), 1.37\left(\mathrm{~s}, 3 \mathrm{H}, \mathrm{CH}_{3}-\mathrm{N}\right)$.

\section{Biological evaluation}

Antimicrobial activity

The antimicrobial activity was evaluated by filter paper disc agar diffusion method ${ }^{(23)}$. For antibacterial studies Hi-media bacteriological nutrient broth ${ }^{(24)}$ and bacteriological nutrient agar were used against Gram-positive Bacillus subtilis, Staphylococcus aureus and Gram-negative Salmonella typhi and Escherichia coli. Antifungal studies were carried out using Sabouraud's dextrose broth and dextrose agar against Aspergillus fumigatus, Aspergillus niger, Alternaria alternate and Penicillum chrysogenum. Dimethyl formamide was used as solvent and also for control studies. The concentration of the compounds taken was $1 \mathrm{mg} \mathrm{ml} l^{-1}$. Norfloxaxin $\left(1 \mathrm{mg} \mathrm{ml}^{-1}\right)$ and clotrimazole $\left(1 \mathrm{mg} \mathrm{ml}^{-1}\right)$ were used as standards for bacterial and fungal studies respectively. The sensitivity of microorganisms to the compounds is identified in the flowing table (Table 2).

Electron-rich nitrogen heterocycles play an important role in diverse biological activities. Introducing a pyrazolone ring ${ }^{(25-26)}$ in place of the $\beta$-lactam 
ring in penicillins and cephalosporins ${ }^{(27)}$ results in enhanced activity. Second nitrogen in the five-membered ring also influences the antibacterial or pharmacokinetic properties ${ }^{(28-30)}$.

The antimicrobial activity of some newly synthesized compounds (3a,b; 4a,b; 5a,b; 6a-e; 7a,d; 8b,c; 9a-c) were tested and the results are shown in Table 2. Evaluation of the new compounds established that compounds $6 \mathrm{a}, 6 \mathrm{~d}, 7 \mathrm{a}, 9 \mathrm{a}$, and $9 \mathrm{c}$ have shown the highest inhibitory activity against Gram-positive bacterium " $B$. subtilis", while compounds 5b, 6a, 6d, 7a, d, 8c, 9a,c have shown the same highest inhibitory activity against Gram-positive bacterium "S. aureus". It was also noticed that compounds $5 \mathrm{~b}, 6 \mathrm{~b}, \mathrm{c}, 6 \mathrm{e}, 7 \mathrm{~d}, 8 \mathrm{~b}$ and $8 \mathrm{~b}$ and compounds $4 \mathrm{~b}, 5 \mathrm{a}, 6 \mathrm{~b}, \mathrm{c}, \mathrm{e}$, $8 \mathrm{~b}$, and $9 \mathrm{~b}$ as well demonstrated antibacterial activity less effective towards Grampositive bacterium "B. subtilis" and "S. aureus," respectively. Compounds 3a, 4a,b, $5 \mathrm{a}, 8 \mathrm{~b}$ and $9 \mathrm{~b}$ were slightly active against the same bacterium, while compounds $3 \mathrm{~b}$ was inactive. For the Gram-negative bacterium. "S. typhi" and "E. coli", compounds $3 \mathrm{a}, 6 \mathrm{a}-\mathrm{d}, 7 \mathrm{a}, 8 \mathrm{~b}, 9 \mathrm{a}$ revealed the highest inhibitory activity, while compounds $3 \mathrm{~b}, 4 \mathrm{a}, \mathrm{b}, 5 \mathrm{a}, \mathrm{b}, 6 \mathrm{~d}, 7 \mathrm{~d}, 8 \mathrm{c}, 9 \mathrm{~b}, \mathrm{c}$ were moderately sensitive against those two organisms, and compound 6a, demonstrated no sensitivity against $E$. coli for the fungi used, A. fumigatus, A. nigen, A. alternata and P. chynsogenum compounds $3 \mathrm{a}, \mathrm{b}, 4 \mathrm{a}, \mathrm{b}, 6 \mathrm{~b}$ revealed no activity towards all of them while compound $9 \mathrm{c}$ was more effective than the other tested compounds. Compounds $6 \mathrm{a}$, c, 7a, d, were slightly active against the tested fungi (c.f. Table 2).

TABLE 2. In vitro antimicrobial activity of some newly synthesized heterocyclic compounds.

\begin{tabular}{|ccccccccc|}
\hline & \multicolumn{2}{c}{ Gram-positive } & \multicolumn{2}{c|}{ Gram-negative } & \multicolumn{5}{c|}{ Fungi } \\
Compd. & $\begin{array}{c}\text { Bacillus } \\
\text { subtilis }\end{array}$ & Staphylococcus & Salmonella & Escherichia & Aspergillus & Aspergillus & Alternaria & Penicillium \\
& aureus & typhi & coli & fumigatus & niger & alternate & chrysogenum \\
\hline 3a & 5 & 5 & 16 & 16 & 0 & 0 & 0 & 0 \\
3b & 0 & 0 & 14 & 12 & 0 & 0 & 0 & 0 \\
$\mathbf{4 a}$ & 5 & 5 & 10 & 10 & 0 & 0 & 0 & 0 \\
$\mathbf{4 b}$ & 10 & 10 & 14 & 12 & 0 & 0 & 0 & 0 \\
$\mathbf{5 a}$ & 10 & 10 & 10 & 10 & 0 & 5 & 5 & 5 \\
$\mathbf{5 b}$ & 14 & 15 & 14 & 12 & 5 & 5 & 12 & 12 \\
$\mathbf{6 a}$ & 18 & 15 & 18 & 0 & 0 & 12 & 12 & 12 \\
$\mathbf{6 b}$ & 12 & 13 & 16 & 16 & 0 & 0 & 0 & 0 \\
$\mathbf{6 c}$ & 14 & 12 & 18 & 16 & 12 & 12 & 13 & 12 \\
$\mathbf{6 d}$ & 18 & 18 & 16 & 12 & 12 & 0 & 0 & 12 \\
$\mathbf{6 e}$ & 14 & 14 & 12 & 14 & 12 & 12 & 10 & 10 \\
$\mathbf{7 a}$ & 18 & 15 & 16 & 16 & 12 & 10 & 12 & 10 \\
$\mathbf{7 d}$ & 14 & 17 & 14 & 14 & 12 & 12 & 0 & 12 \\
$\mathbf{8 b}$ & 10 & 10 & 18 & 18 & 10 & 10 & 10 & 12 \\
$\mathbf{8 c}$ & 15 & 18 & 10 & 10 & 12 & 10 & 10 & 10 \\
$\mathbf{9 a}$ & 15 & 15 & 15 & 15 & 10 & 10 & 10 & 6 \\
$\mathbf{9 b}$ & 10 & 10 & 10 & 10 & 10 & 10 & 10 & 0 \\
$\mathbf{9 c}$ & 17 & 17 & 13 & 12 & 18 & 18 & 17 & 10 \\
\hline
\end{tabular}

where: highly sensitive $=15-20 \mathrm{~mm}$; moderately sensitive $=10-15 \mathrm{~mm}$; slightly sensitive 5-15 mm; slightly sensitive $5-10 \mathrm{~mm}$ and not sensitive $=0 \mathrm{~mm}$. 


\section{References}

1. Lopez, S.E., Rosales, M.E., Canelon, C.E., Valverode, E.A., Narvaez, R.C., Charris, J.E., Giannini, F.A., Enriz, R.D., Carrasco, M., and Zacchino, S., Synthesis and preliminary cytotoxic and antifungal evaluation of some 6-N,Ndialkyl 2-Aryl-4(3H)-quinazolinone derivatives. Heterocycl. Commun, 7(5), 473-480 (2001).

2. Bekhit, A.A., Habbib, N.S. and Bekhit, El.A., Synthesis and antimicrobial evaluation of chalcone and syndrome derivatives of $4(3 \mathrm{H})$-quinazolinone. Boll. Chim. Farm. 140(5), 297-301 (2001).

3. Usifoh, C.O. and Scriba, G.K.E., Synthesis and anticonvulsant activity of acetylenic quinazolinone derivatives. Arch. Pharm. (Weinheim), 333, 261 (2000).

4. Kulcsar, G., Kalai, T., Osz, E., Sar, C.P., Jeko, J., Sumegi, B. and Hideg, K., "The role of akt and mitogen-activated protein kinase systems in the protective effect of poly(ADP-sibose) polymerase inhibition in langendorff perfused and in isoproterenoldamaged rat hearts. Arkivoc, 121 ( 2003).

5. Raffa, D., Daidone, G., Schillaci, D., Maggio, B. and Plescia. F., Synthesis of new 3(3-Phenyl-isoxazol-5-yl) or 3-[(3-Phenyl-isoxazol-5-yl) amino] Substituted 4(3H)quinazolinone derivatives with antineoplastic activity. Pharmazie, 54, 251 (1999).

6. Ahluwalia, V.K. and Chandra, R.J. Miniprint; A convenient synthesis of novel pyrimidine analogues of o-hydroxy chalcones and pyrano [2,3-d] pyrimidines and their biological activities. Chem. Res. 4, 162-163 (2000).

7. Flefel, E.M., Salama, M.A., El-Stahat, M., El-Hashash, M.A. and El-Farargy, A.F., A novel synthesis of some new pyrimidine and thiazolopyrimidine derivatives for anticancer evaluation. Phosphorus, Sulphur and Silicon, 182(8), 1739 (2007).

8. Farag A.M., Ali. K. A.K., Taha M.A.; Abdelrahman S. M.; Abdel-Galil E. and Mohamed, M., "Design, synthesis and structure-activity relationship study of novel pyrazole-based heterocycles as potential antitumor agents. Eur.J. Med. Chem, 45(12), 5887-5989 (2010).

9. Propsavin, M., Torovic, L., Spaic, S., Stankov, S., Kapor, A., Tomic, Z. and Popsavin, V., Synthesis and biological evaluation of new pyrazole- and tetrazolerelated $C$-nucleosides with modified sugar moieties. Tetrahedron, 58(3), 569-580 (2002).

10. Turan-Zitouni, G., Özdemir, A. and Güven, K., Synthesis of some 1-[(N, Ndisubstituted thiocarbamoylthio) acetyl] -3- (2-thienyl) -5- aryl -2-pyrazoline derivatives and investigation of their antibacterial and antifungal activities. Arch Pharm. Med. Chem. 338, 96-104 (2005).

11. Turan-Zitouni, G., Ozdemir. A., Kaplancikli, Z.A., Chevallet, P. and Tunali. Y. Synthesis and antimicrobial activities of some 1-[(N, Ndisubstitutedthiocarbamoylthio) acetyl] - 3,5 - diaryl - 2 - pyrazolines. Phosphorus Sulfur, Silicon Relat. Elem 180, 2717-24 ( 2005). 
12. Niementowski, V., Reaction of anthranilic acid with amide to form 4-oxo- 3, 4dihydro quinazoline. J. Prakt, Chem. 51, 564, Beilestein 24, 143 (1895).

13. Ziegler, E., Steiger, W. and Kappe, Th. Monatsh. Chem. 100, 150-152 (1969).

14. Kaur, R., Bansal, M. and Kaur., B., Synthesis of some new quinazoline derivatives and theoritical studies of their geometrics. Chemical Sciences J. 18 (2011).

15. Murugesan, D., Periyaswamy, S., Erik, D. and Seshaiah, K.S., Synthesis, antiviral and Cytotoxic Activity of 6- Bromo-2, 3- disubstituted-4(3H) quinazolinones. Biol. Pharm. Bull. 26 (9), 1278-1282 (2003).

16. Soliman, F.M., Eslam, I.E., Kassab, R.R.,Souka, L.M. and El-Kady, M.Y., Chem Inform Abstract: A new approach for the synthesis of some benzoxazinone and quinazolinone derivatives. Rev. Roum. Chim. 39(4), 405-411 (1994).

17. Hamidian, H., Tikdari, A.M., and Khabazzadeh, H; Synthesis of new 4(3H)quinazolinone derivatives using -5(4H)-oxazolones. Molecules, 11, 377-382 (2006).

18. Chavadad. B. N., Patel, R. and Vanpuria., S. Study on the antimicrobial activity for normal and multidrug resistant strains for 3-benzensulfonamide substituted quinazolinone derivatives in in-vitro condition. I J PA S, 1 (1), 70-73 (2010).

19. Mosaad, S.M., Mohsen, M. M. and Kassem, N.A., Novel 6,8-dibromo-4 (3H) quinazolinone derivatives of antibacterial and antifungal activities. Eur. J. Med. Chem. 45, 3311-3319 (2010).

20. Misra, R.S., Dwivedi, C. and Parmar, S.S., Synthesis of 2-substituted styryl-6bromo-4-quinazolone 3-(4-benzhydrazides) as possible monoamine oxidase inhibitors. J. Heterocycl. Chem. 17(6), 1337 (1980).

21. Singh, S.P., Parmer, S.S. and Stenberg, V.I., T.K., Carbon-13 nuclear magnetic resonance spectrum of methaqualone. J. Heterocycl. Chem. 15(1), 53 (1978).

22. Still, J.W., Plavac, N., McKinnon, D.M. and Chauhan, M.S., Can. J. Chem. 56, 725 (1978)

23. Marruzella, J.C. and Henry, P.A., synthesis and antibacterial of some 2- amino -4,6diaryl pyrimidine. J. Am. Pharm. Assoc. 47, 471 (1958).

24. National committee for clinical laboratory standard, References method for broth dilution antifungal susceptibility testing of yeast, approval standard. Document M27A National Committee for Clinical Laboratory Standard, Wayne, PA, USA (1997).

25. Junghein, L.N., Sigmund, S.K. and Fisher, J.W., Bicyclic pyrazolidinones, a new class of antibacterial agent based on the $\beta$-lactam model. Tetrahedron Lett. 28(3), 285-92 (1987).

26. Jungheim, L.N., Sigmund, S.K., Jones, N.D and Swartendruber, J.K., Bicyclic pyrazolidinones, steric and electronic effects on antibacterial activity. Tetrahedron Lett. 28(3), 289-92 (1987).

Egypt. J. Chem. 54, No. 1 (2011) 
27. Boyd, D.B., Theoretical and Physicochemical Studies on $\beta$-lactam Antibiotics In $\beta$ lactam Antibiotics, Chemistry and Biology, In: R.B. Morin, and Gorman (Ed.), Vol. 1, Academic Press, New York, pp. 437-45 (1982).

28. Jungheim, L.N., Holmes, R.E., OH, J.L., Ternasky, R.J., Draheim, S.E., Neel, D.A., Stepherd, T.A. and Sigmund, S.K., Abstracts of $26^{\text {th }}$ Interscience Conf. on Antimicrobial Agents \& Chemotherapy Oct. 1988, 23-26, Los Angels, C.A., Paper 240.

29. Ternanskym, R.J. and Draheim, S.E., An efficient synthesis of a new class of synthetic antibacterial agents. Tetrahedron Lett. 31(20), 2805-08 (1990).

30. Ilango., K, Valentina, N. and Been, K.P., Eco-benign mediated versatile synthesis of newer quinazolin-4-(3H)-one clubbed isatin derivatives as potent antimicrobial agents. Int.J.Res.Pharm.Sci. 1(2), 133-138 (2010).

(Received 13/7/2010;

accepted 3/5/2011)

تخليق والنشاط البيولوجى لبعض مشتقات الكوينازولينون الجديدة

نادية طه علي داوود ، ناهد فتح الله عبد الغفار و فكرية محمد احمد سليمان

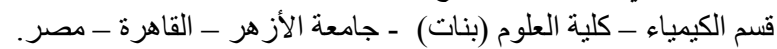

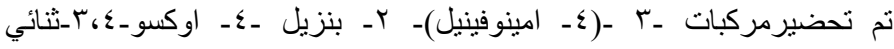

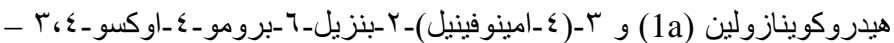

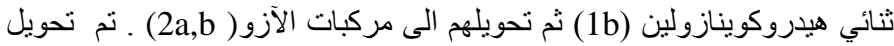

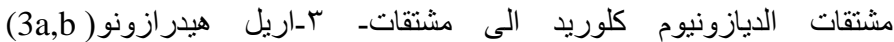

(5a,b) (4a,b)

النشطة مثل استيل اسيتون، ايثيل اسيتو اسيتات ، ايثيل سيانو اسيتات. كذللك تم

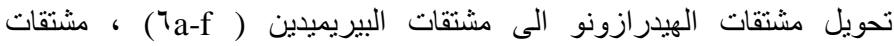

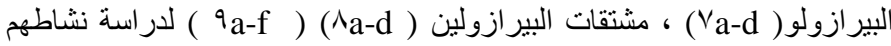

الميكروبي. 